\title{
Selected topics from the 28th International Symposium on Shock Waves, July 17-22, 2011, Manchester, UK
}

\author{
K. Kontis
}

Published online: 25 December 2013

(C) Springer-Verlag Berlin Heidelberg 2013

The current issue is the second out of two issues and contains ten selected articles that were presented at the 28th International Symposium on Shock Waves (ISSW28), held in Manchester, UK, July 17-22, 2011.

The International Symposia on Shock Waves are the definitive meetings of the scientific community devoted to the study and use of the shock-wave phenomena, of all kinds. Started in 1957, they take place every 2 years, at places close to centres of activity in the field.

The call for abstract submission resulted in a total number of 486 abstracts. Each abstract was reviewed by two members of the ISSW28 Scientific Review Committee which consisted of 109 experts in the field of shock wave research. The final programme of the symposium contained 9 plenary lectures and 341 oral and poster contributions. The authors of 28 ISSW28 presentations were invited to submit an archival journal version of their conference papers to Shock Waves Journal. Out of these, 24 papers made it through the peerreview process, with the majority of papers subjected to one or two revisions. All manuscripts were subjected to the regular rigorous review procedure, i.e. they were reviewed by at least two independent referees, each of which is an internationally recognized expert in the field.

Out of 24 accepted papers, 12 articles were included into the first ISSW28 issue of Shock Waves Journal (Volume 23, Number 4, July 2013). Another ten articles appear in the current issue, and the remaining two articles are still in processing and will be included into subsequent regular issues.

I would like to thank the authors who submitted their papers and also, the reviewers whose help was crucial in ensuring the high quality of the papers.
K. Kontis $(\varangle)$

University of Glasgow, Glasgow, UK

e-mail: kostas.kontis@glasgow.ac.uk 